\title{
Anti-tumor peptide AP25 decreases cyclin D1 expression and inhibits MGC-803 proliferation via phospho-extracellular signal-regulated kinase-, Src-, c-Jun N-terminal kinase- and phosphoinositide 3-kinase-associated pathways
}

\author{
JIALIANG HU ${ }^{1 *}$, TAO CHENG ${ }^{1 *}$, LIJUN ZHANG $^{1}$, BEICHENG SUN $^{2}$, LEI DENG $^{2}$ and HANMEI XU ${ }^{1}$ \\ ${ }^{1}$ Key Laboratory of Modern Chinese Medicines, Ministry of Education, China Pharmaceutical University, \\ Nanjing, Jiangsu 211198; ${ }^{2}$ Liver Transplant Center, The First Affiliated Hospital of Nanjing Medical University, \\ Nanjing, Jiangsu 210029, P.R. China
}

Received June 10, 2014; Accepted February 24, 2015

DOI: $10.3892 / \mathrm{mmr} .2015 .3912$

\begin{abstract}
The anti-tumor peptide AP25 is a prototype integrin antagonist, which exhibits anti-angiogenic and anti-tumor activity. The molecular mechanisms by which AP25 inhibits the growth of the MGC-803 gastric carcinoma cell line were investigated in the present study. K-ras specific RNA interference by lentiviral infection was successfully induced in MGC-803 cells [MGC-803 short hairpin (sh)RNA group] and the expression levels of K-ras, phosphorylated extracellular signal-regulated kinase ( $\mathrm{p}-\mathrm{ERK}$ ) and cyclin D1 were observed to be markedly decreased. By contrast, AP25 caused cell cycle arrest of intact MGC-803 cells and decreased p-ERK and cyclin D1 expression levels. Of note, 0.4-3.2 $\mu \mathrm{M}$ AP25 no longer inhibited MGC-803 shRNA growth, indicating that AP25, at such concentrations, exerts its effect mainly through the Ras/Raf/mitogen-activated protein kinase kinase/ERK pathway, whereas at $25 \mu \mathrm{M}, \mathrm{AP} 25$ was able to inhibit MGC-803 shRNA growth. Chemical inhibitors of Src, c-Jun N-terminal kinase (JNK) and phosphoinositide 3-kinase (PI3K) were used to confirm that $25 \mu \mathrm{M}$ AP25 inhibited growth of cells in the MGC-803 shRNA group and activated intracellular signaling pathways with Src, JNK and PI3K as key enzymes. In conclusion, the present study revealed the signal transduction pathways activated by AP25 at low (0.4-3.2 $\mu \mathrm{M})$ or high $(25 \mu \mathrm{M})$ concentrations. It also confirmed that integrins, when interacting with the freely moving ligand AP25 instead of
\end{abstract}

Correspondence to: Professor Hanmei Xu, Key Laboratory of Modern Chinese Medicines, Ministry of Education, China Pharmaceutical University, 18 Zhilan Road, Nanjing, Jiangsu 211198, P.R. China

E-mail: 1037714870@qq.com

*Contributed equally

Key words: integrin, K-ras, RNA interference, cyclin D1 immobilized extracellular matrix glycoproteins, are able to initiate cell signaling via similar pathways as in the latter case but with a reversed effect, to inhibit cell growth.

\section{Introduction}

AP25 is an anti-angiogenic and anti-tumor peptide with molecular targets, including integrin $\alpha 5 \beta 1$ and $\alpha v \beta 3$ (1). AP25 inhibited the growth of the MGC-803, HeLa, HCT116, A549, MDA-MB-435 and MDA-MB-231 cell lines in vitro with half maximal inhibitory concentration values of 8-24 $\mu \mathrm{M}$. AP25 also significantly inhibited the growth of B16F10 in $\mathrm{C} 57 \mathrm{Bl} / 6$ mice and the growth of HCT116 human colon carcinoma cells in severe combined immunodeficient mice (1). This in vivo inhibitory effect is attributed to the inhibition of tumor cell growth and inhibition of angiogenesis (1). The molecular mechanisms by which AP25 inhibits the growth of MGC-803 cells were investigated in the present study.

Cell cycle progression in mammalian cells is strictly regulated by integrin-mediated adhesion to the extracellular matrix and by binding of growth factors to their receptors (2). This regulation is mediated by $\mathrm{G} 1$ phase cyclin-dependent kinases (CDKs), which are downstream of the signaling pathways under the integrated control of integrins and growth factors (3). The regulation of the G1 phase by CDKs is now fairly well understood (4). Of the major components in the G1 phase CDK system (cyclins D and E, CDK4, CDK2 and their inhibitors), integrin/receptor tyrosine kinase (RTK) signaling has been most closely associated with the induction of cyclin D1 (5). The induction of cyclin D1 mRNA has most frequently been attributed to the activation of extracellular signal-regulated kinases (ERKs) (5). In the majority of cases, the activation of the Ras/Raf/mitogen-activated protein kinase kinase/ERK cascade induces cyclin D1 gene expression. Conversely, inhibition of the Ras pathway inhibits cyclin D1 gene expression. By contrast, phosphoinositide 3-kinase (PI3K) is required for the expression and stability of cyclin D1 in certain cell lines $(6,7)$. Furthermore, previous studies have indicated that activation of Rac by integrins is able to increase cyclin D1 levels in 
endothelial cells (8) and overexpression of activated Rac also stimulated cyclin D1 gene expression in fibroblasts (6). In addition to the aforementioned signal transduction pathways, c-Jun $\mathrm{N}$-terminal kinases (JNKs) and p38 mitogen-activated protein kinases also are important downstream of the integrins $(9,10)$.

RNA interference is a process in which double-stranded RNA triggers the degradation of a homologous mRNA. Lentivirus-mediated RNA interference may be used to inhibit the expression of homologous genes in different mammalian cells, in vitro and in vivo. It is an important tool in gene function analysis and gene therapy (11). Ras is activated in cancer cells by the mutation of ras alleles (12) and also by perturbations in RTK signaling (13). The three ras isoforms, H-, N- and K-ras, are ubiquitously expressed in mammalian cells and are able to interact with the same set of effector molecules (14). A previous study confirmed that K-ras recruited Raf-1 to the plasma membrane and activated Raf-1 more efficiently than H-ras, the latter of which was a more potent activator of other signaling pathways, such as the PI3K pathway (14). Furthermore, K-ras mutations occur in $50 \%$ of colon cancer cases and $90 \%$ of pancreatic cancer cases, whereas $\mathrm{N}$ - and H-ras mutations are extremely uncommon (14). Therefore, RNA interference specific for K-ras was performed in order to weaken the signal transduction via the Ras/Raf/MEK/ERK pathway, while simultaneously retaining the basic functions of the remaining ras proteins ( $\mathrm{N}$ - and $\mathrm{H}-\mathrm{ras}$ ), which are important for the maintenance of growth and the survival rate of the infected cells.

In the present study, RNA interference of K-ras was performed to investigate the role of the main signaling pathway downstream of the integrins and the RTKs. Chemical inhibitors of the key enzymes in various signaling pathways downstream of integrins were also included to investigate how integrin engagement resulted in decreased expression levels of cyclin D1.

\section{Materials and methods}

Cell culture, antibodies and reagents. Peptide AP25 was synthesized by GLBiochem Ltd. (Shanghai, China) with a purity of $>95 \%$. The MGC-803 gastric carcinoma cell line as well as 293 cells were purchased from the American Type Culture Collection (Shanghai, China) and maintained in Dulbecco's modified Eagle's medium with $10 \%$ fetal bovine serum (FBS; Invitrogen Life Technologies, Grand Island, NY, USA). The chemical inhibitors of key enzymes in various signaling pathways used in the present study included the Src-selective kinase inhibitor PP1 (cat no. 567809; Merck, Darmstadt, Germany), the PI3K inhibitor wortmannin (cat no. BML-ST415-0001; Biomol, Plymouth Meeting, PA, USA) and the JNK-specific inhibitor SP600125 (cat no. EI-305-0010; Biomol).

Generation of K-ras-specific lentivirus. K-ras-specific lentivirus was prepared as previously described (15). The synthetic transcription short hairpin (sh)RNA template sequences were as follows: forward, 5'-AACGGTCATCCA GTGTTGTCATGCTTTCAAGAGAAGCATGACAACACT GGATGACCTTTTTTC-3' and reverse, 5'-TCGAGAAAA AAGGTCATCCAGTGTTGTCATGCTTCTCTTGAAAGC ATGACAACACTGGATGACCGTT-3'. The sequences were provided by Shanghai Gene Pharma Co., Ltd. (Shanghai, China). The above synthetic and annealed duplexes were sub-cloned between the XhoI and HpaI sites of the plasmid PLentilox3.7 (Invitrogen Life Technologies) to form the expression plasmid PLentilox3.7-shRNA, which was used to transform Escherichia coli DH5 $\alpha$. The bacteria were spread on a lysogeny broth plate containing ampicillin (Hyclone, Logan, UT, USA). The positive colonies were selected and plasmids were identified by enzyme digestion and sequencing as described previously (15).

Confluent 293 cells were cultured in a six-well culture plate to $70-80 \%$ confluency. The recombinant lentivirus was generated from 293 cells co-transfected with calcium phosphate precipitation of plasmids: i) PLentilox-shRNA, and ii) pCMV $\Delta$ R8.91, a plasmid expressing the human immunodeficiency virus-1 gag/pol, tat and rev genes required for efficient lentivirus production, and iii) a plasmid expressing the vesicular stomatitis virus envelope glycoprotein (G), pLR-VSV-G (Invitrogen Life Technologies, Carlsbad, CA, USA), at 15:15:7.5 $\mu \mathrm{g} /$ plate. Cells transfected with empty PLentilox3.7 were used as the control. After $72 \mathrm{~h}$, the viral supernatant was collected and concentrated 500 -fold by ultra-high speed centrifugation at $35,000 \mathrm{x} \mathrm{g}$, at $4^{\circ} \mathrm{C}$ for $2 \mathrm{~h}$. The viral titer was determined to be $1 \times 10^{7}$ transducing units $/ \mathrm{ml}$. The viral stocks were stored frozen at $-80^{\circ} \mathrm{C}$ until usage.

Infection of MGC-803 cells with K-ras-specific lentivirus. A total of $0.05 \mathrm{ml}\left(3 \times 10^{6}\right.$ cells $\left./ \mathrm{ml}\right)$ MGC- 803 cells were seeded into each well of a six-well plate and cultured to $50 \%$ confluence. Viral infection was initiated by addition of $0.9 \mathrm{ml}$ viral stock solution, $0.1 \mathrm{ml}$ complete culture medium and $6 \mu 1$ polybrene (Invitrogen Life Technologies, Grand Island, NY, USA). After $6 \mathrm{~h}$, the viral solution was removed and $2 \mathrm{ml}$ fresh medium with $10 \%$ FBS was added. After $48 \mathrm{~h}$, the cells were observed under a fluorescence microscope (Olympus DP72; Olympus, Tokyo, Japan). The efficiency of infection was determined by the percentage of cells exhibiting green fluorescent protein expression. The MGC-803 cells successfully infected with K-ras-specific or control virus were termed MGC-803 shRNA or MGC-803 NC.

Preparation of protein samples. MGC-803, MGC-803 shRNA and MGC-803 NC cells were washed with ice-cold phosphate-buffered saline (PBS) three times. A total of $300 \mu 1$ ice-cold cell lysis buffer (radioimmunoprecipitation assay buffer; Hyclone) with $1 \%$ phenylmethylsulfonyl fluoride and $1 \%$ phosphatase inhibitor was added to each $24-\mathrm{cm}^{2}$ culture flask. The cells were scraped off the flask quickly with a cell scraper. The cell lysate was collected and preserved on ice for $20 \mathrm{~min}$ to complete lysis. The supernatant was collected by centrifugation at $8,000 \times \mathrm{g}$ for $10 \mathrm{~min}$ at $4^{\circ} \mathrm{C}$ for protein concentration analysis using a bicinchoninic acid assay kit from Tiangen Biotech Co., Ltd. (Beijing, China).

Western blot analysis. A total of $100 \mu \mathrm{g}$ total protein in the whole cell lysate from MGC-803, MGC-803 shRNA and MGC-803 NC cells were subjected to a $12 \%$ SDS-PAGE, followed by blotting with specific antibodies. The protein bands were visualized with an enhanced chemiluminescence plus (ECL+) substrate (Tanon Science and Technology Co., 
Ltd., Shanghai, China). The primary antibodies consisted of a mouse anti-K-ras monoclonal antibody (OP-24; Calbiochem, Darmstadt, Germany; 1:100), a rabbit anti-p-ERK polyclonal antibody (9101; Cell Signaling Technology, Danvers, MA, USA; 1:500), a rabbit anti-ERK polyclonal antibody (9102; Cell Signaling Technology; 1:500), a rabbit anti-cyclin D-1 monoclonal antibody (2922; Cell Signaling Technology; $1: 1,000)$ and a mouse anti- $\beta$-actin monoclonal antibody (TA-09; ZSBG-Bio; Beijing, China; 1:1,000). The membranes were incubated with the primary antibodies overnight at $4^{\circ} \mathrm{C}$. Gray value analysis was performed with ImageJ software version 1.46 (National Institutes of Health, Bethesda, MD, USA) and the results were expressed as a comparison of the gray value of a protein band with that of the corresponding $\beta$-actin band.

Cell cycle analysis. MGC-803 cells were growth-arrested by contact inhibition for $24 \mathrm{~h}$. A total of $200 \mu \mathrm{l}\left(6 \times 10^{6} \mathrm{cells} / \mathrm{ml}\right)$ cells were added to each well of a six-well plate in the presence of $0,3.2$ or $50 \mu \mathrm{M}$ AP25. After $12 \mathrm{~h}$, the cells were harvested and fixed in ice-cold ethanol. The fixed cells were dehydrated at $4^{\circ} \mathrm{C}$ for $30 \mathrm{~min}$ in PBS and were stained with propidium iodine (5 $\mu \mathrm{g} / \mathrm{ml}$; Takara Bio, Inc., Otsu, Japan). The cells were then analyzed using a BD FACSCalibur flow cytometer (BD Biosciences, Mountain View, CA, USA).

Cell proliferation assays. A total of $100 \mu \mathrm{l}\left(2 \times 10^{4}\right.$ cells $\left./ \mathrm{ml}\right)$ MGC-803 or MGC-803 shRNA cells were seeded into each well of a 96-well plate. After $12 \mathrm{~h}$, AP25 or chemical inhibitors at $15 \mu \mathrm{M}$ PP1, $10 \mu \mathrm{M} \mathrm{SP} 600125$ and $30 \mu \mathrm{M}$ wortmannin dissolved in complete cell medium were added. For each concentration there were five repeat wells. After $48 \mathrm{~h}$, cell proliferation was detected with an MTT assay at a detection wavelength of $570 \mathrm{~nm}$ and reference wavelength of $630 \mathrm{~nm}$. The proliferation inhibitory effect of AP25 was calculated as $\left(A_{\text {negative control }}-A_{\text {drug }}\right) / A_{\text {negative control }} \times 100 \%$.

Statistical analysis. All values are expressed as the mean \pm standard deviation. All statistical analyses were performed using the statistical software package SPSS 17.0 (SPSS, Inc., Chicago, IL, USA). P $<0.05$ was considered to indicate a statistically significant difference and $\mathrm{P}<0.01$ was considered to indicate a highly significant difference between values.

\section{Results}

$K$-ras interference in MGC-803 cells by lentiviral infection. $\mathrm{K}$-ras-specific lentiviruses were generated and verified using gel electrophoresis (Fig. 1). Successful infection of MGC-803 cells was performed. Under a fluorescence microscope, $>90 \%$ MGC-803 shRNA cells (Fig. 2A) and MGC-803 NC cells (Fig. 2B) exhibited marked fluorescence, indicating a successful infection and target gene transcription. Using western blot analysis, K-ras protein expression in MGC-803 shRNA cells was significantly decreased, whereas that in the MGC-803 NC cells exhibited no substantial change (Fig. 2C and E). Furthermore, the expression of p-ERK, the important kinase downstream of ras, and cyclin D1 decreased significantly in MGC-803 shRNA cells, whereas that in MGC-803

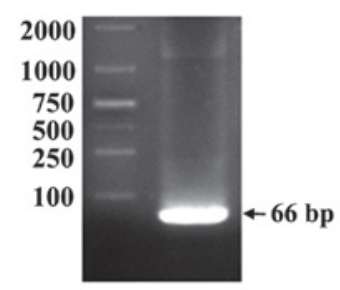

B

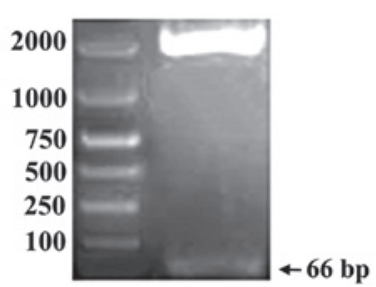

Figure 1. shRNA synthesis and PLentilox3.7-shRNA generation. (A) Agarose gel electrophoresis of chemically synthesized shRNA following heating and annealing. The bright band indicates a large quantity of DNA at the point corresponding to the product length. (B) Agarose gel electrophoresis of target fragments following $\mathrm{Hpal}$ and Xhol digestion of PLentilox3.7-shRNA that was collected from the transformed Escherichia coli DH5 $\alpha$. shRNA, short hairpin RNA.

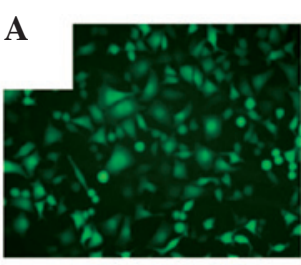

MGC-803 shRNA

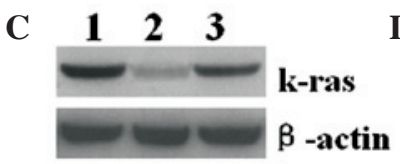

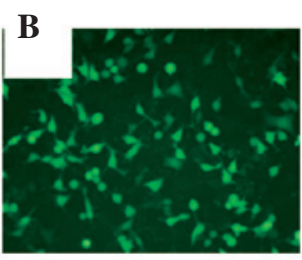

MGC-803 NC
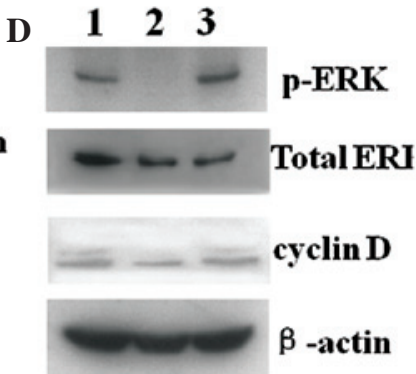

E
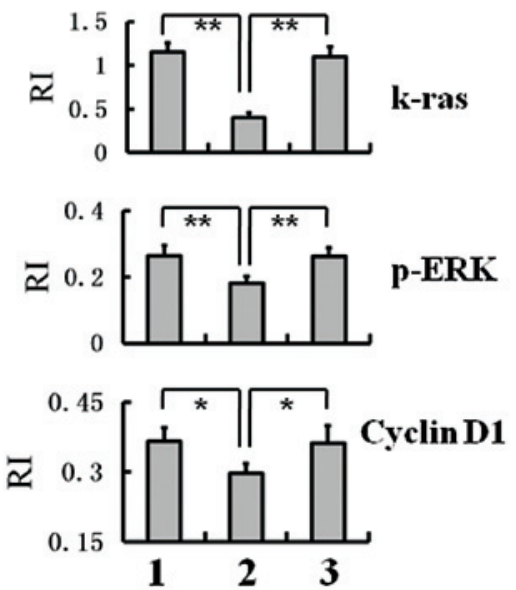

Figure 2. Decreased expression levels of K-ras, p-ERK and cyclin D1 in MGC-803 shRNA cells. (A) Fluorescence microscopy image of MGC-803 cells infected with K-ras-specific lentiviruses (MGC-803 shRNA; magnification, x200). (B) Fluorescence microscopy image of MGC-803 cells infected with control lentiviruses (MGC-803 NC; magnification, x200). Western blot analysis of (C) K-ras and (D) p-ERK and cyclin D1 expression levels in MGC-803, MGC-803 shRNA and MGC-803 NC cells. (E) Gray value analysis of the protein bands in $\mathrm{C}$ and $\mathrm{D}$ to compare the relative protein expression levels of K-ras, $\mathrm{p}-\mathrm{ERK}$ and cyclin $\mathrm{D} 1\left(^{* *} \mathrm{P}<0.01\right)$. RI was obtained by comparison of the gray value of a protein band with that of the corresponding $\beta$-actin band. Groups: 1 , intact MGC-803; 2, MGC-803 shRNA; 3 , MGC-803 NC. shRNA, short hairpin RNA; p-ERK, phosphorylated extracellular signal-regulated kinase; RI, relative intensity; NC, negative control. 
A

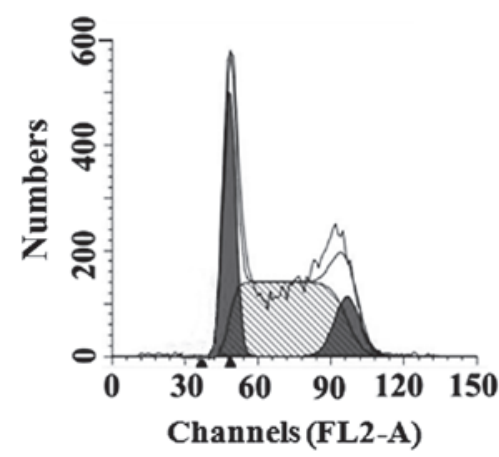

C

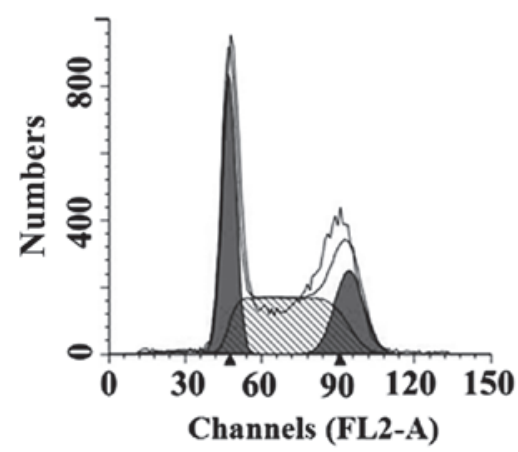

B

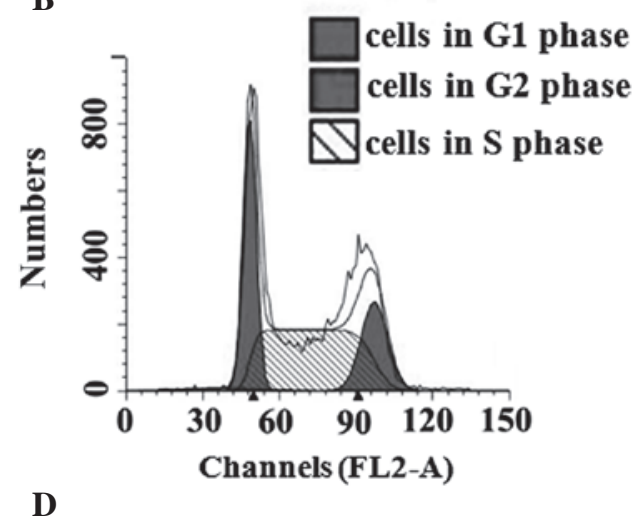

D

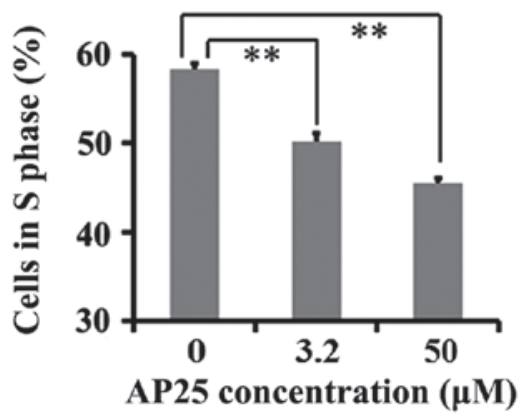

Figure 3. AP25 inhibits intact MGC-803 cell growth via cell cycle arrest. (A-C) Flow cytometric analysis of the percentage of MGC-803 cells in S phase following treatment with (A) $0 \mu \mathrm{M}$, (B) $3.2 \mu \mathrm{M}$ and (C) $50 \mu \mathrm{M}$ AP25. Cells were treated with AP25 for $12 \mathrm{~h}$ and collected for cell cycle analysis following staining with propidium iodide. (D) AP25 dose-dependently caused cell cycle arrest of intact MGC-803 cells. The percentage of cells in S phase were expressed as the mean \pm standard deviation of 3 repetitions $(" \mathrm{P}<0.01)$.

$\mathrm{NC}$ cells remained almost identical to that in the intact cells (Fig. 2D and E). These results indicated that K-ras interference reduced the levels of signaling of the Ras/Raf/MEK/ERK pathway in MGC-803 cells and the level of expression of cyclin D1 was also decreased.

AP25 inhibits MGC-803 cell growth by decreasing $p$-ERK and cyclin D1 levels. Cell cycle analysis was performed on intact MGC-803 cells and as shown in Fig. 3, AP25 treatment significantly decreased the percentage of intact MGC-803 cells in $\mathrm{S}$ phase after a $12 \mathrm{~h}$ incubation in complete medium.

Using MTT assays, AP25 treatment was revealed to inhibit intact MGC-803 cell growth at low $(0.4$ to $3.2 \mu \mathrm{M})$ and high concentrations (6.3 to $100 \mu \mathrm{M})$. At a concentration of $100 \mu \mathrm{M}$, AP25 treatment almost completely inhibited MGC-803 cell growth after a 48-h incubation (Fig. 4A).

AP25 contains a sequence termed ES-2, comprising amino acids $60-70$ of endostatin (1). ES-2 covers one of the two active domains of endostatin to interact with integrin $\alpha 5 \beta 1$ and induce the inhibitory effect of endostatin on angiogenesis (16). It has been observed that endostatin causes G1 arrest of endothelial cells (17) and inhibits human umbilical vein endothelial cell (HUVEC) growth by decreasing the expression levels of cyclin D1 within the cells (18). Importantly, endostatin was unable to arrest cyclin D1-overexpressing endothelial cells, suggesting that cyclin D1 is a critical target for the action of endostatin (18). It was also demonstrated that through interactions with free extracellular ligands, integrins interact with growth factor receptors by interfering with the
Ras/Raf/ERK/cyclin D1 pathway, which is an important signal transduction pathway downstream of growth factor receptors (3). The expression levels of cyclin D1 and p-ERK within intact MGC-803 cells were evaluated following AP25 treatment. As shown in Fig. 4B and C, AP25 decreased the expression levels of cyclin D1 and p-ERK in a dose-dependent manner in intact MGC-803 cells. Together with the data in Fig. 3, it was revealed that AP25 inhibited intact MGC-803 cell growth and decreased cyclin D1 expression via the Ras/Raf/MEK/ERK pathway.

MTT assays were also performed on MGC-803 shRNA cells. As shown in Fig. 4D, at low concentrations $(0.4$ to $3.2 \mu \mathrm{M})$, AP25 exhibited no inhibitory effect on MGC-803 shRNA cell growth. As K-ras interference in MGC-803 shRNA cells decreased the expression levels of p-ERK and cyclin D1, it is possible that AP25 was not able to further suppress cell signaling in the Ras/Raf/ERK/cyclin D1 pathway to further inhibit the growth of MGC-803 shRNA cells. However, at high concentrations (25 to $100 \mu \mathrm{M}$ ), AP25 exerted a marked inhibition of MGC-803 shRNA cell growth, which indicated that at high concentrations, AP25 used alternative signaling pathways to inhibit MGC-803 shRNA cell growth.

Src, JNK and PI3K are key enzymes in the signaling pathways activated by $25 \mu M$ AP25. To further investigate which signaling pathways mediated the inhibitory effect of AP25 at high concentrations, chemical inhibitors of Src (PP1), JNK (SP600125), and PI3K (wortmannin) were included in the MTT assay and the western blot analysis. As shown in Fig. 5A, 
$\mathbf{A}$
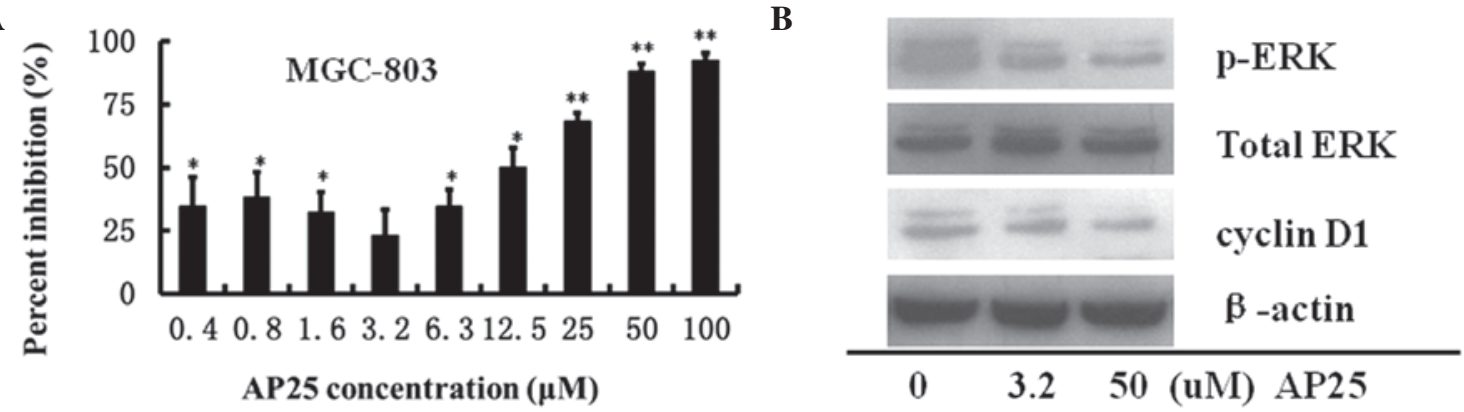

C

AP25 concentration $(\mu \mathrm{M})$

D
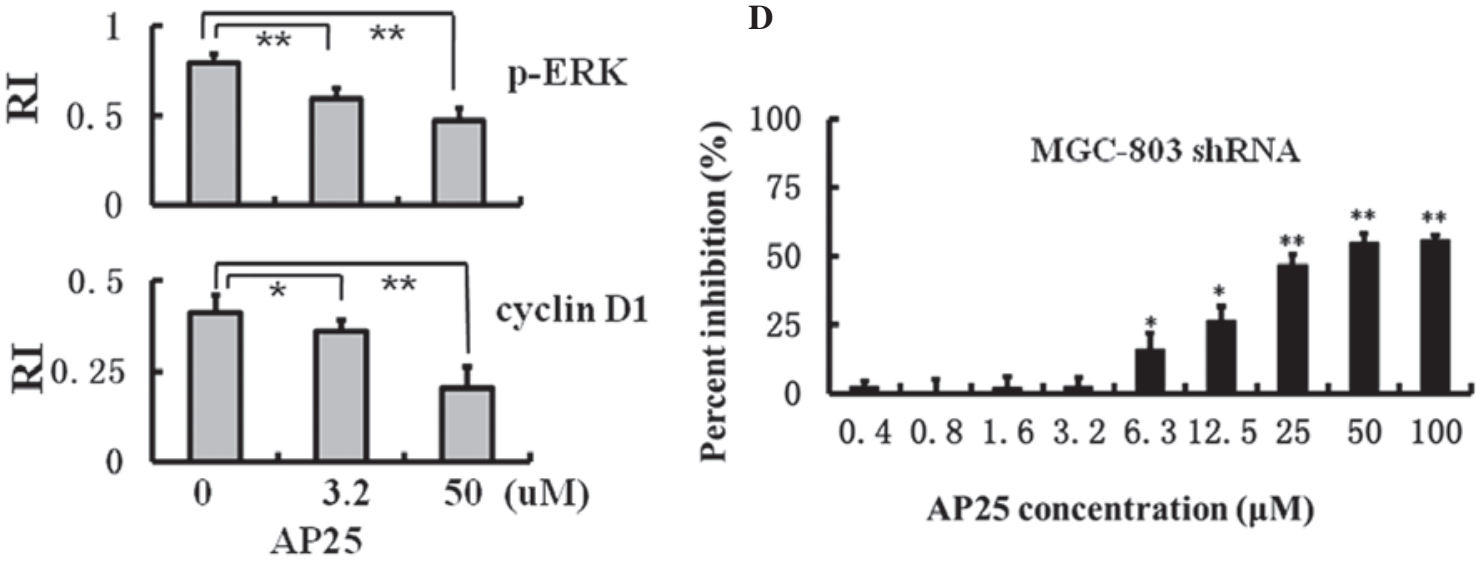

Figure 4. Effect of AP25 on the growth of intact MGC-803 and MGC-803 shRNA cells. (A) MTT analysis of the inhibitory effect of AP25 on MGC-803 cell growth at low $(0.4-3.2 \mu \mathrm{M})$ and high $(6.3-100 \mu \mathrm{M})$ concentrations. The percentage of MGC-803 growth inhibition was expressed as mean \pm standard deviation of 5 repetitions. P-values were calculated based on the comparison between the growth of AP25 treated cells and AP25 untreated cells. ( $\mathrm{P}<0.05$; $\left.{ }^{* *} \mathrm{P}<0.01\right)$ (B) Western blot analysis of p-ERK, total ERK and cyclin D1 expression following AP25 treatment in intact MGC-803 cells. (C) Gray value analysis of the results in B. (D) MTT analysis of the inhibitory effect of AP25 on the growth of MGC-803 shRNA cells. The percentage of MGC-803 shRNA growth inhibition was expressed as mean \pm standard deviation of 5 repetitions. P-values were calculated based on the comparison between the growth of AP25 treated cells and AP25 untreated cells. ( $\mathrm{P}<0.05$; $\left.^{* *} \mathrm{P}<0.01\right)$. shRNA, short hairpin RNA; p-ERK, phosphorylated extracellular signal-regulated kinase; RI, relative intensity.

A

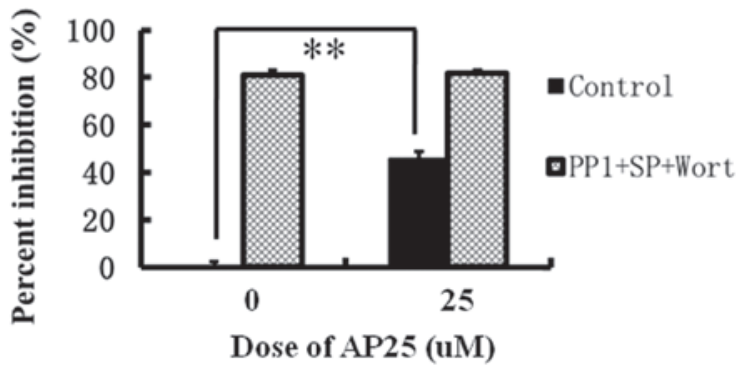

B

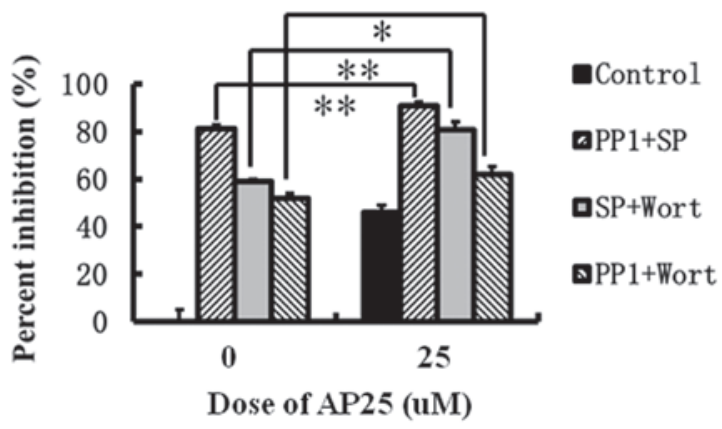

Figure 5. Src, JNK and PI3K are key enzymes in the signaling pathways activated following treatment with $25 \mu \mathrm{M}$ AP25. (A) $25 \mu \mathrm{M}$ AP25 did not further increase the combinatorial inhibition of MGC-803 shRNA cell growth by $15 \mu \mathrm{M} \mathrm{PP} 1,10 \mu \mathrm{M}$ SP600125 and $30 \mu \mathrm{M}$ wortmannin. (B) $25 \mu \mathrm{M}$ AP25 further increased the combinatorial inhibition of MGC-803 shRNA cells by two of the three inhibitors $\left({ }^{*} \mathrm{P}<0.05 ;{ }^{* *} \mathrm{P}<0.01 ; \mathrm{n}=5\right.$ for all experiments) shRNA, short hairpin RNA; JNK, c-Jun N-terminal kinase; PI3K, phosphoinositide 3-kinase; Wort, wortmannin; SP, SP600125. in the MTT assay, $25 \mu \mathrm{M}$ AP25 exhibited a $45 \%$ inhibition of MGC-803 shRNA cell growth. Quantities of $15 \mu \mathrm{M}$ PP1, $10 \mu \mathrm{M}$ SP600125 and $30 \mu \mathrm{M}$ wortmannin together caused an $80 \%$ inhibition of MGC-803 shRNA cell growth, whereas the additional presence of $25 \mu \mathrm{M}$ AP25 did not further increase the combinatorial inhibition, indicating that the signaling pathways activated by AP25 at high concentrations are within the scope of the Src-, JNK- and PI3K-associated pathways, in addition to the Ras/Raf/ERK pathway. Furthermore, the combinatorial inhibition of MGC-803 shRNA cell growth by two of the three enzyme inhibitors in the presence or absence of $25 \mu \mathrm{M}$ AP25 were compared. A significant difference was identified in the combinatorial inhibition by PP1 and SP600125 in the presence (90\%) or absence $(80 \%)$ of $25 \mu \mathrm{M}$ AP25 $(\mathrm{P}<0.01)$ (Fig. 5B), indicating that an alternative signaling pathway existed downstream of that activated by $25 \mu \mathrm{M}$ AP25 in MGC-803 shRNA cells in which PI3K is important. Similarly, the significant difference in the combinatorial inhibition of MGC-803 shRNA growth by SP600125 and wortmannin in the presence $(80 \%)$ and absence $(60 \%)$ of $25 \mu \mathrm{M}$ AP25 (P<0.01) (Fig. 5B) indicated that the Src-associated signaling existed downstream of the pathway initiated by $25 \mu \mathrm{M}$ AP25. The significant difference identified between the combinatorial inhibition by PP1 and wortmannin in the presence (65\%) and absence (55\%) of $25 \mu \mathrm{M}$ AP25 $(\mathrm{P}<0.05)$ confirmed that the signaling pathway with JNK as an important factor also existed downstream of that initiated by $25 \mu \mathrm{M}$ AP25 (Fig. 5B). 


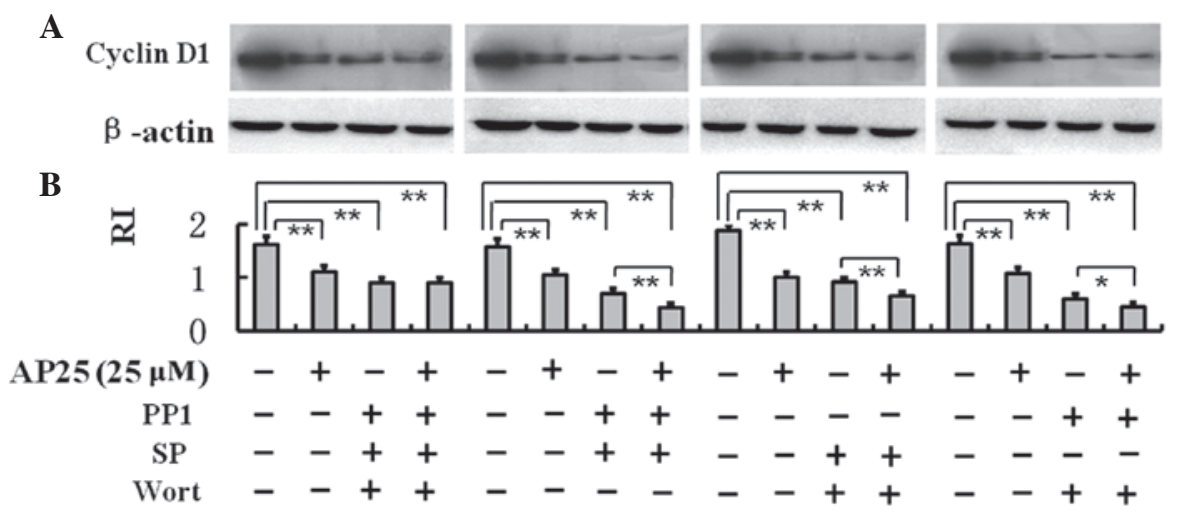

Figure 6. Western blot analysis of cyclin D1 expression in MGC-803 shRNA cells. (A) Images of western blot analysis of cyclin D1 expression levels in MGC-803 shRNA cells following treatment with $25 \mu \mathrm{M} \mathrm{AP} 25$ and/or the combination of $15 \mu \mathrm{M} \mathrm{PP} 1,10 \mu \mathrm{M}$ SP600125 and $30 \mu \mathrm{M}$ Wort. (B) Gray value analysis of the images in $\mathrm{A}$. The treatment conditions are indicated under the columns $\left({ }^{* *} \mathrm{P}<0.01\right)$. shRNA, short hairpin RNA; RI, relative intensity; Wort, wortmannin.

Western blot analysis of cyclin D1 expression levels within MGC-803 shRNA cells confirmed the results of the MTT assay. In Fig. 6A and B, treatment with $25 \mu \mathrm{M}$ AP25 decreased the expression levels of cyclin D1. The combinatorial inhibition of Src, JNK and PI3K also decreased the expression levels of cyclin D1, whereas the combinatorial inhibition by the three inhibitors in the presence of $25 \mu \mathrm{M}$ AP25 did not further decrease the expression levels of cyclin D1. By contrast, co-treatment with $25 \mu \mathrm{M}$ AP25 further decreased cyclin D1 expression in addition to the combinatorial effect of PP1 and SP100625, the combinatorial effect of SP100625 and wortmannin and the combinatorial effect of PP1 and wortmannin.

\section{Discussion}

Anti-angiogenic reagents often have differential effects at low or high concentrations, such as the anti-angiogenic peptide HM-3, which is formed by binding of the RGD sequence to the C-terminus of ES-2. HM-3 inhibits HUVEC migration at $5 \mu \mathrm{M}$, whereas at $38 \mu \mathrm{M}$, it promotes HUVEC migration (19). Similarly, AP25 inhibited HUVEC migration at $0.2 \mu \mathrm{M}$, whereas at $12.8 \mu \mathrm{M}$, it promoted HUVEC migration. A possible reason for this may be that at higher concentrations, HM-3 and AP25 activate alternative signaling pathways and the crosstalk of the various signaling pathways results in a reversal of the cellular behavior. According to the experimental results of the present study, AP25 at low concentrations inhibited MGC-803 cell growth mainly through the Ras/Raf/ERK/cyclin D1 pathway, whereas at high concentrations, AP25 activated alternative signaling pathways. As AP25 exhibited an inhibitory effect on MGC-803 cell growth, key signaling pathways and key enzymes may be confirmed only in a negative way, such as blocking the Ras/Raf/MEK/ERK pathway by K-ras interference, which, at low concentrations, reduced the inhibitory effect of AP25 on MGC-803 shRNA cell growth. In addition, key signaling pathways and key enzymes were confirmed only after examining the effects of high doses of AP25; following the omission of one out of three enzyme inhibitors, high concentrations of AP25 further increased the combinatorial inhibitory effect of the other chemical inhibitors.
As previously reported, the Ras/Raf/MEK/ERK pathway is the main pathway regulating cell cycle progression via regulation of cyclin D1 expression (5). This was confirmed in the present study, which showed that AP25 caused cell cycle arrest in untreated and treated MGC-803 cells and decreased p-ERK and cyclin D1 expression levels. K-ras interference was successfully induced and it was confirmed that the Ras/Raf/MEK/ERK pathway was the main signal transduction pathway at low concentrations of AP25 $(0.4$ to $3.2 \mu \mathrm{M})$ to inhibit MGC-803 cell growth. However, at $25 \mu \mathrm{M}$, alternative pathways were activated, indicating that only after K-ras interference and Src, JNK and PI3K inhibition by chemical inhibitors, $25 \mu \mathrm{M}$ AP25 no longer inhibited MGC-803 shRNA cell growth. It is noteworthy that AP25 is a freely moving ligand for integrins. The conventional role of integrins is to promote cell growth and the survival rate when interacting with immobilized extracellular matrix glycoproteins. However, when interacting with freely moving extracellular ligands, such as AP25, they accumulate intracellular signaling molecules and generate signal transduction pathways to inhibit cell growth (20). The target signaling pathways selected in the present study were those that were well investigated and downstream of integrins interacting with immobilized ligands (21). It was observed that when interacting with freely moving ligands, integrins generated intracellular signaling with a similar set of key molecules, but with a different effect. The proximal mechanisms of integrin signaling remain to be elucidated and require further investigation to explain how promoting effect of integrin agonists on cell growth switches to an inhibitory effect with a similar set of intracellular key molecules. This may improve the current understanding of the mechanism by which integrin antagonists exhibit their anti-tumor effects in clinical trials.

\section{Acknowledgements}

The present study was supported by 863 High-Technology Development Planning (grant no. SQ2011SF11B02030), the National Natural Science Foundation of China (grant no. 81301902), the National Science and Technology Major Projects of New Drugs (grant nos. 2012ZX09103301-004 and 2014ZX09508007) and the International Projects of Scientific 
Cooperation and Communication (grant no. 2012DFG32000) in China.

\section{References}

1. Yin R, Zheng H, Xi T and Xu HM: Effect of RGD-4C position is more important than disulfide bonds on antiangiogenic activity of RGD-4C modified endostatin derived synthetic polypeptide. Bioconjug Chem 21: 1142-1147, 2010.

2. Stoker M, O'Neill C, Berryman S and Waxman V: Anchorage and growth regulation in normal and virus-transformed cells. Int J Cancer 3: 683-693, 1968.

3. Schwartz MA and Assoian RK: Integrins and cell proliferation: regulation of cyclin-dependent kinases via cytoplasmic signaling pathways. J Cell Sci 114: 2553-2560, 2001.

4. Sherr CJ and Roberts JM: CDK inhibitors: positive and negative regulators of G1 phase progression. Genes Dev 13: 1501-1512, 1999

5. Roovers K and Assoian RK: Integrating the MAP kinase signal into the G1 phase cell cycle machinery. Bioessays 22: 818-826, 2000.

6. Gille $\mathrm{H}$ and Downward J: Multiple ras effector pathways contribute to G(1) cell cycle progression. J Biol Chem 274: 22033-22040, 1999.

7. Takuwa N, Fukui Y and Takuwa Y: Cyclin D1 expression mediated by phosphatidylinositol 3-kinase through mTOR-p70(S6K)-independent signaling in growth factor-stimulated NIH $3 \mathrm{~T} 3$ fibroblasts. Mol Cell Biol 19: 1346-1358, 1999.

8. Huang S, Chen CS and Ingber DE: Control of cyclin D1, p27(Kip1), and cell cycle progression in human capillary endothelial cells by cell shape and cytoskeletal tension. Mol Biol Cell 9: 3179-3193, 1998.

9. Verma G, Bhatia H and Datta M: Gene expression profiling and pathway analysis identify the integrin signaling pathway to be altered by IL-1 $\beta$ in human pancreatic cancer cells: role of JNK Cancer Lett 320: 86-95, 2012.
10. Niederlechner S, Baird C and Wischmeyer PE: P38MAP kinase, but not phosphoinositol-3 kinase, signal downstream of glutamine-mediated fibronectin-integrin signaling after intestinal injury. Nutr J 12: 88, 2013

11. Lois C, Refaeli Y, Qin XF and Van Parijs L: Retroviruses as tools to study the immune system. Curr Opin Immunol 13: 496-504, 2001.

12. Bos JL: ras oncogenes in human cancer: a review. Cancer Res 49 : 4682-4689, 1989.

13. Shields JM, Pruitt K, McFall A, Shaub A and Der CJ: Understanding Ras: 'it ain't over 'til it's over'. Trends Cell Biol 10: $147-154,2000$.

14. Yan J, Roy S, Apolloni A, Lane A and Hancock JF: Ras isoforms vary in their ability to activate Raf-1 and phosphoinositide 3-kinase. J Biol Chem 273: 24052-24056, 1998.

15. Deng L, Li G, Xi L, et al: Hepatitis B virus inhibition in mice by lentiviral vector mediated short hairpin RNA. BMC Gastroenterol 9: 73, 2009.

16. Olsson AK, Johansson I, Akerud $\mathrm{H}$, et al: The minimal active domain of endostatin is a heparin-binding motif that mediates inhibition of tumor vascularization. Cancer Res 64: 9012-9017, 2004.

17. Dhanabal M, Volk R, Ramchandran R, Simons $M$ and Sukhatme VP: Cloning, expression, and in vitro activity of human endostatin. Biochem Biophys Res Commun 258: 345-352, 1999.

18. Hanai J, Dhanabal M, Karumanchi SA, et al: Endostatin causes G1 arrest of endothelial cells through inhibition of cyclin D1. J Biol Chem 277: 16464-16469, 2002.

19. Xu H, Pan L, Ren Y, et al: RGD-modified angiogenesis inhibitor HM-3 dose: dual function during cancer treatment. Bioconjug Chem 22: 1386-1393, 2011.

20. Garmy-Susini B and Varner JA: Roles of integrins in tumor angiogenesis and lymphangiogenesis. Lymphat Res Biol 6: 155-163, 2008.

21. Cox D, Brennan M and Moran N: Integrins as therapeutic targets: Lessons and opportunities. Nat Rev Drug Discov 9: 804-820, 2010. 